\title{
Multiple Solutions for Nonlinear Doubly Singular Three-Point Boundary Value Problems with Derivative Dependence
}

\author{
R. K. Pandey and A. K. Barnwal \\ Department of Mathematics, Indian Institute of Technology, Kharagpur 721302, India \\ Correspondence should be addressed to R. K. Pandey, rkp@maths.iitkgp.ernet.in \\ Received 25 May 2012; Accepted 12 July 2012 \\ Academic Editor: Yuji Liu
}

Copyright (c) 2012 R. K. Pandey and A. K. Barnwal. This is an open access article distributed under the Creative Commons Attribution License, which permits unrestricted use, distribution, and reproduction in any medium, provided the original work is properly cited.

We study the existence of multiple nonnegative solutions for the doubly singular threepoint boundary value problem with derivative dependent data function $-\left(p(t) y^{\prime}(t)\right)^{\prime}=$ $q(t) f\left(t, y(t), p(t) y^{\prime}(t)\right), 0<t<1, y(0)=0, y(1)=\alpha_{1} y(\eta)$. Here, $p \in C[0,1] \cap C^{1}(0,1]$ with $p(t)>0$ on $(0,1]$ and $q(t)$ is allowed to be discontinuous at $t=0$. The fixed point theory in a cone is applied to achieve new and more general results for existence of multiple nonnegative solutions of the problem. The results are illustrated through examples.

\section{Introduction}

In this paper, we consider the following three-point boundary value problem of SturmLiouville type:

$$
-\left(p(t) y^{\prime}(t)\right)^{\prime}=q(t) f\left(t, y(t), p(t) y^{\prime}(t)\right), \quad 0<t<1,
$$

with boundary conditions

$$
y(0)=0, \quad y(1)=\alpha_{1} y(\eta)
$$

where $0<\alpha_{1}<(h(1)) /(h(\eta))$ and $h(t)=\int_{0}^{t}(1 / p(x)) d x$.

Throughout this paper, we assume the following conditions on the functions $p(t), q(t)$, and $f\left(t, y, p y^{\prime}\right)$ :

(E1) $p \in C[0,1] \cap C^{1}(0,1]$ with $p(t)>0$ on $(0,1]$ and $1 / p \in L^{1}(0,1)$; 
(E2) $q(t)>0, q$ is not identically zero on $[0,1]$ and $q \in L^{1}(0,1)$;

(E3) $f \in C([0,1] \times[0, \infty) \times \mathbb{R},[0, \infty))$ and $f$ is not identically zero.

Note that condition (E2) allows $q(t)$ be discontinuous at $t=0$, and if $p(0)=0$, then the differential equation (1.1) is called doubly singular [1].

Nonlocal boundary value problem have variety of applications in the area of applied mathematics and physical sciences. The design of a large size bridge with multipoint supports can be considered as an application of these types of boundary value problem [2]. Some more applications can be found in [3-5] and the references therein. Recently, motivated by the wide application of boundary value problems in physical and applied mathematics, the study of multipoint boundary value problems has received increasing interest (see $[2,6-12]$ and the references therein).

Nonsingular multipoint boundary value problems have been extensively studied in literature, see [13-16] for derivative dependent data function $f(t, y, z)$ and $[8,10,12]$ for derivative independent data function $f(t, y, z)=f(t, y)$.

Some attention has been devoted to singular multipoint boundary value problems (see $[17,18]$ and the references therein). When $p(t)=1$ and $q(t) f(t, y, z)$ may have singularity at $t=0, t=1, y=0$ and $z=0$, differential equation (1.1) with boundary conditions $y^{\prime}(0)=0, y(1)=\alpha_{1} y(\eta)$ is considered by Chen et al. [17] and Agarwal et al. [18]. Chen et al. proved the existence of at least one positive solution while Agarwal et al. established that this problem may have at least two positive solutions and also may have no positive solutions under some conditions on $q(t)$ and $f(t, y, z)$.

Bai and Ge [19] have generalized the Leggett-Williams fixed point theory and applied to

$$
\begin{gathered}
y^{\prime \prime}+q(t) f\left(t, y, y^{\prime}\right)=0, \quad t \in(0,1), \\
y(0)=0, \quad y(1)=0,
\end{gathered}
$$

to achieve at least three positive solutions of the two-point boundary value problem.

In this work, we consider the problem (1.1)-(1.2) with unbounded coefficient of $y^{\prime}$ along with singularity in the data function $f(t, y, z)$.

Existence of nonnegative solution(s) of the problem (1.1)-(1.2) may be established either directly or by reducing the problem to

$$
y^{\prime \prime}+q(t) f\left(t, y, y^{\prime}\right)=0
$$

and applying the existing results. But direct consideration of the problem provides better results, especially as the order of singularity increases. This may be demonstrated by the following simple linear three-point boundary value problem:

$$
\begin{gathered}
-\left(t^{r} y^{\prime}(t)\right)^{\prime}=t^{r-1}\left[2(r+1) t-\frac{5}{4} r\right], \quad 0.5 \leq r<1, t \in(0,1), \\
y(0)=0, \quad y(1)=y\left(\frac{1}{4}\right) .
\end{gathered}
$$


The problem (1.5) can be reduced to the following boundary value problem:

$$
\begin{gathered}
-v^{\prime \prime}(x)=\frac{1}{(1-r)^{2}} x^{(2 r-1) /(1-r)}\left[2(r+1) x^{1 /(1-r)}-\frac{5}{4} r\right], \quad x \in(0,1), \\
v(0)=0, \quad v(1)=v\left[\left(\frac{1}{4}\right)^{(1-r)}\right],
\end{gathered}
$$

by change of variable $x=(1-r) \int_{0}^{t} \tau^{-r} d \tau=t^{1-r}$.

Now we apply the result (Theorem 4.2) of this work to the problem (1.5) and conclude that the problem has at least one nonnegative solution $y(t)$ with

$$
\sup _{t \in[0,1]}|y(t)| \leq \frac{3}{2}\left[\frac{3(1-r)}{4-4^{r}}\right]^{(1-r) / r}\left[\frac{3}{4} r+2\right]
$$

Further, for $p(t)=1$, Theorems 4.2 and 4.3 may be regarded as extension of Theorem 3.1 in [19] for three-point singular boundary value problem. Now applying Theorem 4.2 with $p(t)=1$ to the reduced problem (1.6), we get that the problem (1.5) has at least one nonnegative solution $y(t)$ with

$$
\sup _{t \in[0,1]}|y(t)|=\sup _{t \in[0,1]}|v(t)| \leq \frac{3}{4-4^{r}}\left[\frac{3(1-r)}{4-4^{r}}\right]^{(1-r) / r}\left[\frac{3}{4} r+2\right] .
$$

Now as $r$ approaches to one, that is, the order of singularity increases, the upper bound for $\sup _{t \in[0,1]}|y(t)|$ in (1.8) approaches to $\infty$ while in (1.7) approaches to 4.125 , which can be seen from Figure 1 . As smaller upper bound for $\sup _{t \in[0,1]}|y(t)|$ will enable to find nonnegative solution(s) faster and hence will be helpful in constructing efficient numerical algorithms to find multiple nonnegative solutions, thus it is justified to consider the singular problem directly. A detailed working is given in Example 5.1.

In this work, we are concerned with existence of multiple nonnegative solutions of the three-point doubly singular boundary value problem (1.1)-(1.2). To achieve this, we use generalized Leggett-Williams fixed point theorem established by Bai and Ge [19].

For this purpose, we first establish certain properties of Green's function of the corresponding homogeneous boundary value problem. Then fixed point theorem of functional type (generalized Leggett-Williams fixed point theorem) is applied to yield multiple nonnegative solutions for the boundary value problem (1.1)-(1.2).

We organize this work as follows. In Section 2, we present some definitions and basic results required for this work. Section 3 deals with nonnegativity of Green's function and some basic properties. Section 4 is devoted to existence of at least one and three or odd number of nonnegative solutions. In Section 5, we demonstrate the results through examples.

\section{Background and Definitions}

The proof of main results is based on fixed point theorem of functional type in a cone given by Bai and Ge [19], which deals with three fixed points of completely continuous nonlinear 


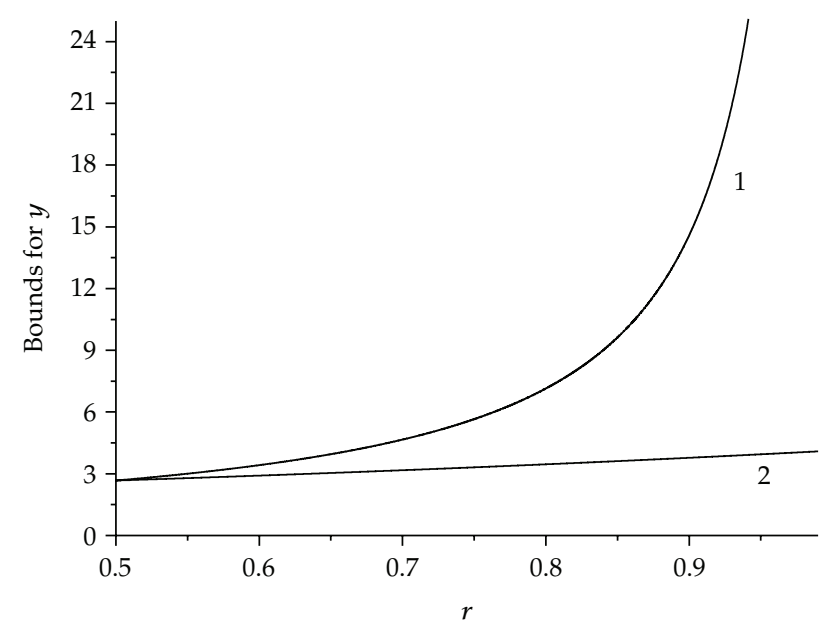

(1) When singular problem is reduced to regular problem

(2) When singular problem is solved directly

Figure 1: Variation of bounds for $y$ in both cases.

operators defined in a cone of an ordered Banach space. In this section, we provide some background material from the theory of cone in Banach spaces to make the paper selfcontained.

Definition 2.1. A subset $D$ of Banach space $E$ is said to be retract of $E$ if $\exists$ a continuous map $r: E \rightarrow D$ such that $r(x)=x$ for every $x \in D$.

Corollary 2.2. Every close convex set of a Banach space is a retract of Banach space.

Definition 2.3. Let $E$ be a Banach space, $P \subset E$ is nonempty convex, closed set, $P$ is said to be cone provided that

(1) $\lambda u \in P$ for all $\lambda \geq 0, u \in P$, and

(2) $u \in P,-u \in P$ implies $u=0$.

Note. From Corollary 2.2, a cone $P$ of Banach Space $E$ is retract of $E$.

Definition 2.4. A subset $R$ of Banach space $X$ is called relatively compact if $\bar{R}$ (closure of $R$ ) is compact.

Definition 2.5. Consider two Banach spaces $X$ and $Y$, a subset $\Omega$ of $X$, and a map $T: \Omega \rightarrow Y$. Then $T$ is said to be completely continuous operator if

(1) $T$ is continuous, and

(2) $T$ maps bounded subset of $\Omega$ into relatively compact sets. 
Definition 2.6. The map $\alpha$ is said to be a nonnegative continuous convex functional on $P$ provided that $\alpha: P \rightarrow[0, \infty)$ is continuous and

$$
\alpha(t x+(1-t) y) \leq t \alpha(x)+(1-t) \alpha(y)
$$

for all $x, y \in P$ and $0 \leq t \leq 1$. Similarly, the map $\gamma$ is said to be a nonnegative continuous concave functional on $P$ provided that $\gamma: P \rightarrow[0, \infty)$ is continuous and

$$
\gamma(t x+(1-t) y) \geq \operatorname{tr}(x)+(1-t) \gamma(y)
$$

for all $x, y \in P$, and $0 \leq t \leq 1$.

Definition 2.7. Suppose $\alpha, \beta: P \rightarrow[0, \infty)$ are two continuous convex functionals satisfying

$$
\|x\| \leq M \max \{\alpha(x), \beta(x)\}, \quad \text { for } x \in P,
$$

where $M$ is positive constant, and

$$
\Omega=\{x \in P: \alpha(x)<r, \beta(x)<L\} \neq \phi \quad \text { for } r>0, L>0 .
$$

From (2.3) and (2.4), $\Omega$ is a bounded nonempty open subset of $P$.

Definition 2.8. Let $r>a>0, L>0$ be given constants, $\alpha, \beta: P \rightarrow[0, \infty)$ two nonnegative continuous convex functionals satisfying (2.3) and (2.4), and $\psi$ a nonnegative continuous concave functional on the cone $P$. Then bounded convex sets are defined as

$$
\begin{gathered}
P\left(\alpha^{r}, \beta^{L}\right)=\{x \in P: \alpha(x)<r, \beta(x)<L\}, \\
\bar{P}\left(\alpha^{r}, \beta^{L}\right)=\{x \in P: \alpha(x) \leq r, \beta(x) \leq L\}, \\
P\left(\alpha^{r}, \beta^{L}, \psi_{a}\right)=\{x \in P: \alpha(x)<r, \beta(x)<L, \psi(x)>a\}, \\
\bar{P}\left(\alpha^{r}, \beta^{L}, \psi_{a}\right)=\{x \in P: \alpha(x) \leq r, \beta(x) \leq L, \psi(x) \geq a\} .
\end{gathered}
$$

Theorem 2.9 (see [20]). Let X be retract of real Banach space E. Then for every bounded relatively open subset $U$ of $X$ and every completely continuous operator $T: \bar{U} \rightarrow X$ which has no fixed point on $\partial U$ (relative to $X)$, there exists an integer $i(T, U, X)$ such that if $i(T, U, X) \neq 0$, then $T$ has at least one fixed point in $U$. Moreover, $i(T, U, X)$ is uniquely defined.

Theorem 2.10 (see [20]). Let $E$ be Banach space, $X$ retract of $E, X_{1}$ a bounded convex retract of $X$, and $U \subset X$ nonempty open subset, such that $U \subset X_{1}$. If $T: X_{1} \rightarrow X$ is completely continuous, $T\left(X_{1}\right) \subset X_{1}$, such that there is no fixed point of $T$ in $X_{1} \backslash U$, then $i(T, U, X)=1$.

Theorem 2.11 ((see [19]) (fixed point theorem of functional type)). Let E be Banach space, $P \subset E$ a cone, and $c \geq b>a>d>0, L_{2} \geq L_{1}>0$ given constants. Assume that $\alpha, \beta$ 
are nonnegative continuous convex functionals on $P$ such that (2.3) and (2.4) are satisfied. $\psi$ is a nonnegative continuous concave functional on $P$ such that $\psi(x) \leq \alpha(x)$ for all $x \in \bar{P}\left(\alpha^{c}, \beta^{L_{2}}\right)$ and let $T: \bar{P}\left(\alpha^{c}, \beta^{L_{2}}\right) \rightarrow \bar{P}\left(\alpha^{c}, \beta^{L_{2}}\right)$ be a completely continuous operator. Suppose that

(1) $\left\{x \in \bar{P}\left(\alpha^{b}, \beta^{L_{2}}, \psi_{a}\right): \psi(x)>a\right\} \neq \Phi$ and $\psi(T x)>a$ for $x \in \bar{P}\left(\alpha^{b}, \beta^{L_{2}}, \psi_{a}\right)$,

(2) $\alpha(T x)<d, \beta(T x)<L_{1}$ for all $x \in \bar{P}\left(\alpha^{d}, \beta^{L_{1}}\right)$,

(3) $\psi(T x)>a$ for all $x \in \bar{P}\left(\alpha^{c}, \beta^{L_{2}}, \psi_{a}\right)$ with $\alpha(T x)>b$.

Then $T$ has at least three fixed points $x_{1}, x_{2}, x_{3} \in \bar{P}\left(\alpha^{c}, \beta^{L_{2}}\right)$ such that

$$
\begin{gathered}
x_{1} \in P\left(\alpha^{d}, \beta^{L_{1}}\right), \quad x_{2} \in\left\{x \in \bar{P}\left(\alpha^{c}, \beta^{L_{2}}, \psi_{a}\right): \psi(x)>a\right\}, \\
x_{3} \in \bar{P}\left(\alpha^{c}, \beta^{L_{2}}\right) \backslash\left(\bar{P}\left(\alpha^{c}, \beta^{L_{2}}, \psi_{a}\right) \cup \bar{P}\left(\alpha^{d}, \beta^{L_{1}}\right)\right) .
\end{gathered}
$$

\section{Some Preliminary Results}

In this section, we construct the Green's function and establish some properties, required to establish the main results in Section 4.

Lemma 3.1. The Green's function for the following boundary value problem:

$$
\left.\begin{array}{r}
-\left(p(t) y^{\prime}(t)\right)^{\prime}=0 \\
\text { B.C. }:=y(0)=0, \quad y(1)=\alpha_{1} y(\eta)
\end{array}\right\}, \quad t \in(0,1)
$$

is given by

$$
G(t, s)= \begin{cases}G_{1}(t, s), & 0 \leq s \leq \min \{t, \eta\}<1 \\ G_{2}(t, s), & 0 \leq t \leq s \leq \eta \\ G_{3}(t, s), & \eta \leq s \leq t \leq 1 \\ G_{4}(t, s), & 0<\max \{\eta, t\} \leq s \leq 1\end{cases}
$$

Here

$$
\begin{gathered}
G_{1}(t, s)=\frac{h(s)}{\delta}\left[\delta-h(t)+\alpha_{1} h(t)\right], \\
G_{2}(t, s)=\frac{h(t)}{\delta}\left[\delta-h(s)+\alpha_{1} h(s)\right], \\
G_{3}(t, s)=\frac{1}{\delta}\left[h(t)\left\{\alpha_{1} h(\eta)-h(s)\right\}+\delta h(s)\right], \\
G_{4}(t, s)=\frac{h(t)}{\delta}[h(1)-h(s)], \\
\delta=h(1)-\alpha_{1} h(\eta)>0, \quad h(t)=\int_{0}^{t} \frac{1}{p(x)} d x, \quad 0<\alpha_{1}<\frac{h(1)}{h(\eta)} .
\end{gathered}
$$


Proof. Consider the following linear differential equation:

$$
-\left(p(t) y^{\prime}(t)\right)^{\prime}=q(t) F(t)
$$

where $F \in C([0,1],[0, \infty))$. Integrating the above differential equation twice first from $t$ to 1 and then from 0 to $t$, changing the order of integration, and applying the boundary conditions, we get

$$
\begin{aligned}
y(t)= & \int_{0}^{t} h(s) q(s) F(s) d s+h(t) \int_{t}^{1} q(s) F(s) d s \\
& +\frac{h(t)}{\delta}\left[\alpha_{1} \int_{0}^{\eta} h(s) q(s) F(s) d s-\int_{0}^{1} h(s) q(s) F(s) d s+\alpha_{1} h(\eta) \int_{\eta}^{1} q(s) F(s) d s\right] .
\end{aligned}
$$

For $t \in[0, \eta], y(t)$ can be written as

$$
\begin{aligned}
y(t)= & \int_{0}^{t} \frac{h(s)}{\delta}\left[\delta-h(t)+\alpha_{1} h(t)\right] q(s) F(s) d s \\
& +\int_{t}^{\eta} \frac{h(t)}{\delta}\left[\delta-h(s)+\alpha_{1} h(s)\right] q(s) F(s) d s+\int_{\eta}^{1} \frac{h(t)}{\delta}[h(1)-h(s)] q(s) F(s) d s,
\end{aligned}
$$

or

$$
y(t)=\int_{0}^{t} G_{1}(t, s) q(s) F(s) d s+\int_{t}^{\eta} G_{2}(t, s) q(s) F(s) d s+\int_{\eta}^{1} G_{4}(t, s) q(s) F(s) d s .
$$

Similarly, for $t \in[\eta, 1], y(t)$ can be written as

$$
\begin{aligned}
y(t)= & \int_{0}^{\eta} \frac{h(s)}{\delta}\left[\delta-h(t)+\alpha_{1} h(t)\right] q(s) F(s) d s \\
& +\int_{\eta}^{t} \frac{1}{\delta}\left[h(t)\left\{\alpha_{1} h(\eta)-h(s)\right\}+\delta h(s)\right] q(s) F(s) d s+\int_{t}^{1} \frac{h(t)}{\delta}[h(1)-h(s)] q(s) F(s) d s,
\end{aligned}
$$

or

$$
y(t)=\int_{0}^{\eta} G_{1}(t, s) q(s) F(s) d s+\int_{\eta}^{t} G_{3}(t, s) q(s) F(s) d s+\int_{t}^{1} G_{4}(t, s) q(s) F(s) d s .
$$

From (3.7) and (3.9), we may write

$$
y(t)=\int_{0}^{1} G(t, s) q(s) F(s) d s
$$


where $G(t, s)$ is given in the lemma. It is easy to see that $G(t, s)$ satisfies all the properties of Green's function. Hence $G(t, s)$ is the Green's function for the boundary value problem (3.1).

Lemma 3.2. The Green's function $G(t, s)$ satisfies the following properties:

(i) $\max _{t \in[0,1]} p(t)(\partial G(t, s) / \partial t)<\infty$,

(ii) $G(t, s) \geq 0$ for all $(t, s) \in\{[0,1] \times[0,1]\}$,

(iii) there exist a constant $\lambda$ in $(0,1)$ such that $\min _{t \in[\eta, 1]} G(t, s) \geq \lambda \max _{t \in[0,1]} G(t, s)$ for $s \in$ $[0,1]$, where

$$
\lambda= \begin{cases}\min \left\{\frac{\alpha_{1}[h(1)-h(\eta)]}{h(1)-\alpha_{1} h(\eta)}, \frac{\alpha_{1} h(\eta)}{h(1)}\right\}, & 0<\alpha_{1} \leq 1 \\ \min \left\{\frac{h(1)-\alpha_{1} h(\eta)}{\alpha_{1}[h(1)-h(\eta)]}, \frac{h(\eta)}{\alpha_{1} h(1)}\right\}, & 1 \leq \alpha_{1}<\frac{h(1)}{h(\eta)}\end{cases}
$$

Proof. (i)

$$
p(t) \frac{\partial G(t, s)}{\partial t}= \begin{cases}\frac{h(s)}{\delta}\left[\alpha_{1}-1\right], & 0 \leq s \leq \min \{t, \eta\}<1 ; \\ \frac{1}{\delta}\left[\delta-h(s)-\alpha_{1} h(s)\right], & 0 \leq t \leq s \leq \eta ; \\ \frac{1}{\delta}\left[\alpha_{1} h(\eta)-h(s)\right], & \eta \leq s \leq t \leq 1 ; \\ \frac{1}{\delta}[h(1)-h(s)], & 0<\max \{\eta, t\} \leq s \leq 1 .\end{cases}
$$

Since $p(t)(\partial G(t, s) / \partial t)$ is independent of $t$, therefore $\max _{t \in[0,1]} p(t)(\partial G(t, s) / \partial t)<\infty$.

(ii) For $t<\eta, \alpha_{1}[h(t)-h(\eta)]>h(1) / h(\eta)[h(t)-h(\eta)]$ and

$$
G_{1}(t, s) \geq \frac{1}{\delta} h(s) h(t)\left[\frac{h(1)}{h(\eta)}-1\right] \geq 0
$$

it is easy to show that $G_{1}(t, s) \geq 0$, for $t \geq \eta$.

Next we show $G_{2}(t, s) \geq 0, G_{3}(t, s) \geq 0$ and $G_{4}(t, s) \geq 0$ as follows:

$$
\begin{aligned}
& G_{2}(t, s) \geq \frac{h(t)}{\delta h(\eta)}[h(s)(h(1)-h(\eta))] \geq 0 ; \\
& G_{3}(t, s)=\frac{1}{\delta}\left[h(s)\{h(1)-h(t)\}+\alpha_{1} h(\eta)\{h(t)-h(s)\}\right] \geq 0, \\
& G_{4}(t, s)=\frac{h(t)}{\delta}[h(1)-h(s)] \geq 0 .
\end{aligned}
$$

Thus $G(t, s) \geq 0$ for all $(t, s) \in\{[0,1] \times[0,1]\}$. 
(iii) We prove the inequality for the following cases:

(a) $s \in[0, \eta]$ and (b) $s \in[\eta, 1]$,

(a) for $s \in[0, \eta]$, we further divide this case in two parts as follows.

(1) When $0<\alpha_{1} \leq 1$, (2) when $1 \leq \alpha_{1}<h(1) / h(\eta)$.

Case 1 (For $0<\alpha_{1} \leq 1$ ). It is easy to see that

$$
G_{1}(t, s)=\frac{h(s)}{\delta}\left[\left(h(1)-\alpha_{1} h(\eta)\right)+h(t)\left(\alpha_{1}-1\right)\right]
$$

implies

$$
\max _{t \in[0,1]} G_{1}(t, s)=\frac{h(s)}{\delta}\left[h(1)-\alpha_{1} h(\eta)\right], \quad \min _{t \in[\eta, 1]} G_{1}(t, s)=\frac{\alpha_{1} h(s)}{\delta}[h(1)-h(\eta)] .
$$

Next,

$$
\begin{aligned}
G_{2}(t, s) & =\frac{h(t)}{\delta}\left[h(1)-\alpha_{1} h(\eta)+h(s)\left(\alpha_{1}-1\right)\right] \\
& \leq \frac{h(s)}{\delta}\left[h(1)-\alpha_{1} h(\eta)\right], \quad \text { as } h(t) \leq h(s) .
\end{aligned}
$$

Thus for $0<\alpha_{1} \leq 1$,

$$
\max _{t \in[0,1]} G(t, s)=\frac{h(s)}{\delta}\left[h(1)-\alpha_{1} h(\eta)\right], \quad \min _{t \in[\eta, 1]} G(t, s)=\frac{\alpha_{1} h(s)}{\delta}[h(1)-h(\eta)] .
$$

Case 2 (For $\left.1 \leq \alpha_{1}<h(1) / h(\eta)\right)$. It is easy to see that

$$
\max _{t \in[0,1]} G_{1}(t, s)=\frac{\alpha_{1} h(s)}{\delta}[h(1)-h(\eta)], \quad \min _{t \in[\eta, 1]} G_{1}(t, s)=\frac{h(s)}{\delta}[h(1)-h(\eta)] .
$$

Next,

$$
\begin{aligned}
G_{2}(t, s) & =\frac{h(t)}{\delta}\left[h(1)-\alpha_{1} h(\eta)+h(s)\left(\alpha_{1}-1\right)\right] \\
& \leq \frac{\alpha_{1} h(s)}{\delta}[h(1)-h(\eta)], \quad \text { as } h(t) \leq h(s) \leq h(\eta) .
\end{aligned}
$$

Thus for $1 \leq \alpha_{1}<h(1) / h(\eta)$,

$$
\max _{t \in[0,1]} G(t, s)=\frac{\alpha_{1} h(s)}{\delta}[h(1)-h(\eta)], \quad \min _{t \in[\eta, 1]} G(t, s)=\frac{h(s)}{\delta}[h(1)-h(\eta)] .
$$


Combining (3.18) and (3.21), we may write for $s \in[0, \eta]$,

$$
\begin{gathered}
\max _{t \in[0,1]} G(t, s)= \begin{cases}\frac{h(s)}{\delta}\left[h(1)-\alpha_{1} h(\eta)\right], & 0<\alpha_{1} \leq 1, \\
\frac{\alpha_{1} h(s)}{\delta}[h(1)-h(\eta)], & 1 \leq \alpha_{1}<\frac{h(1)}{h(\eta)},\end{cases} \\
\min _{t \in[\eta, 1]} G(t, s)=\left\{\begin{array}{cc}
\frac{\alpha_{1} h(s)}{\delta}[h(1)-h(\eta)], & 0<\alpha_{1} \leq 1, \\
\frac{h(s)}{\delta}[h(1)-h(\eta)], & 1 \leq \alpha_{1}<\frac{h(1)}{h(\eta)} .
\end{array}\right.
\end{gathered}
$$

(b) For $s \in[\eta, 1]$. For this case, $G_{3}(t, s)$ and $G_{4}(t, s)$ are considered. From (3.2), it can be easily seen that for $s \in[\eta, 1]$,

$$
\max _{t \in[0,1]} G(t, s)=\frac{h(1)}{\delta}[h(1)-h(s)], \quad \min _{t \in[\eta, 1]} G(t, s)=\frac{h(\eta)}{\delta}[h(1)-h(s)] .
$$

Thus from (3.22) and (3.23), we get

$$
\begin{aligned}
& \max _{t \in[0,1]} G(t, s)=\left\{\begin{array}{cl}
s \in[0, \eta]: \begin{cases}\frac{h(s)}{\delta}\left[h(1)-\alpha_{1} h(\eta)\right], & 0<\alpha_{1} \leq 1, \\
\frac{\alpha_{1} h(s)}{\delta}[h(1)-h(\eta)], & 1 \leq \alpha_{1}<\frac{h(1)}{h(\eta)},\end{cases} \\
s \in[\eta, 1]:\left\{\frac{h(1)}{\delta}[h(1)-h(s)],\right. & 0<\alpha_{1}<\frac{h(1)}{h(\eta)} .
\end{array}\right. \\
& \min _{t \in[\eta, 1]} G(t, s)=\left\{\begin{array}{cl}
s \in[0, \eta]: \begin{cases}\frac{\alpha_{1} h(s)}{\delta}[h(1)-h(\eta)], & 0<\alpha_{1} \leq 1, \\
\frac{h(s)}{\delta}[h(1)-h(\eta)], & 1 \leq \alpha_{1}<\frac{h(1)}{h(\eta)}\end{cases} \\
s \in[\eta, 1]:\left\{\frac{h(\eta)}{\delta}[h(1)-h(s)],\right. & 0<\alpha_{1}<\frac{h(1)}{h(\eta)} .
\end{array}\right.
\end{aligned}
$$

From (3.24),

$$
\min _{t \in[\eta, 1]} G(t, s) \geq \begin{cases}\min \left\{\frac{\alpha_{1}[h(1)-h(\eta)]}{h(1)-\alpha_{1} h(\eta)}, \frac{\alpha_{1} h(\eta)}{h(1)}\right\} \max _{t \in[0,1]} G(t, s), & 0<\alpha_{1} \leq 1 \\ \min \left\{\frac{h(1)-\alpha_{1} h(\eta)}{\alpha_{1}[h(1)-h(\eta)]}, \frac{h(\eta)}{\alpha_{1} h(1)}\right\} \max _{t \in[0,1]} G(t, s), & 1 \leq \alpha_{1}<\frac{h(1)}{h(\eta)}\end{cases}
$$


Consequently, setting

$$
\lambda= \begin{cases}\min \left\{\frac{\alpha_{1}[h(1)-h(\eta)]}{h(1)-\alpha_{1} h(\eta)}, \frac{\alpha_{1} h(\eta)}{h(1)}\right\}, & 0<\alpha_{1} \leq 1, \\ \min \left\{\frac{h(1)-\alpha_{1} h(\eta)}{\alpha_{1}[h(1)-h(\eta)]}, \frac{h(\eta)}{\alpha_{1} h(1)}\right\}, & 1 \leq \alpha_{1}<\frac{h(1)}{h(\eta)},\end{cases}
$$

there holds

$$
\min _{t \in[\eta, 1]} G(t, s) \geq \lambda \max _{t \in[0,1]} G(t, s)
$$

It can be easily seen that $0<\lambda<1$. This completes the proof.

\section{Existence of Multiple Nonnegative Solutions}

Let $X=C[0,1] \cap C^{2}(0,1]$ be endowed with ordering $x \leq y$ if $x(t) \leq y(t)$ for all $t \in[0,1]$ and $\|x\|=\max \left\{\|x\|_{1},\left\|x^{\prime}\right\|_{1}\right\}$, where

$$
\begin{gathered}
\|x\|_{1}=\sup _{t \in[0,1]}|x(t)|, \\
\left\|x^{\prime}\right\|_{1}=\sup _{t \in(0,1]}\left|p(t) x^{\prime}(t)\right| .
\end{gathered}
$$

Let $E=\{x: x \in X,\|x\|<\infty\}$ be bounded subset of $X$. $E$ is Banach Space.

Now define a cone $P \subset E$ as

$$
P=\left\{x \in E: x(t) \geq 0, \min _{t \in[\eta, 1]} x(t) \geq \lambda \max _{t \in[0,1]} x(t),\left(p(t) x^{\prime}(t)\right)^{\prime} \leq 0\right\}
$$

The boundary value problem (1.1)-(1.2) has a solution $y(t)$ if and only if $y(t)$ solves the following operator equation:

$$
y(t)=T y(t)
$$

where the operator $T: P \rightarrow P$ is given by

$$
(T y)(t)=\int_{0}^{1} G(t, s) q(s) f\left(s, y(s), p(s) y^{\prime}(s)\right) d s, \quad 0 \leq t \leq 1
$$

Here $G(t, s)$ is the Green's function of the problem (3.1) defined in Lemma 3.1.

Lemma 4.1. Let (E1)-(E3) hold, then the operator $T: P \rightarrow P$ is well defined and is completely continuous. 
Proof. First we show that the operator $T$ is well defined. For this, we take $y \in P$. From (E2), (E3), and $G(t, s) \geq 0$, it follows that $T y(t) \geq 0$.

Now applying Lemma 3.2, we get

$$
\begin{aligned}
\min _{t \in[\eta, 1]} T y(t) & =\min _{t \in[\eta, 1]} \int_{0}^{1} G(t, s) q(s) f\left(s, y(s), p(s) y^{\prime}(s)\right) d s, \\
\min _{t \in[\eta, 1]} T y(t) & \geq \lambda \int_{0}^{1} \max _{t \in[0,1]} G(t, s) q(s) f\left(s, y(s), p(s) y^{\prime}(s)\right) d s, \\
& =\lambda \max _{t \in[0,1]} \int_{0}^{1} G(t, s) q(s) f\left(s, y(s), p(s) y^{\prime}(s)\right) d s, \\
& =\lambda \max _{t \in[0,1]}(T y)(t) .
\end{aligned}
$$

It is easy to show that $\left(p(t) T y^{\prime}(t)\right)^{\prime} \leq 0$. Thus $T$ is well defined.

We now show that $T$ is completely continuous. Let $\left\{y_{n}\right\}$ be a sequence in $P$ and $y_{0} \in P$ with $\lim _{n \rightarrow \infty} y_{n}=y_{0}$. Then, there exists a constant $k_{1}>0$ such that $\left\|y_{n}\right\|<k_{1}$ for all $n \in$

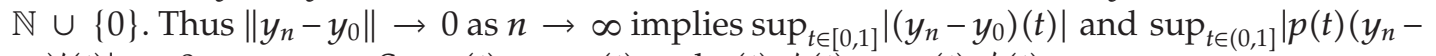
$\left.y_{0}\right)^{\prime}(t) \mid \rightarrow 0$ as $n \rightarrow \infty$. So $y_{n}(t) \rightarrow y_{0}(t)$ and $p(t) y_{n}^{\prime}(t) \rightarrow p(t) y_{0}^{\prime}(t)$ as $n \rightarrow \infty$.

Since $f$ is continuous on $\left\{[0,1] \times\left[0, k_{1}\right] \times\left[-k_{1}, k_{1}\right]\right\}$, so

$$
\begin{aligned}
\left|T y_{n}(t)-T y_{0}(t)\right| & =\left|\int_{0}^{1} G(t, s) q(s)\left[f\left(s, y_{n}(s), p(s) y_{n}^{\prime}(s)\right)-f\left(s, y_{0}(s), p(s) y_{0}^{\prime}(s)\right)\right] d s\right| \\
& \longrightarrow 0 \text { as } n \longrightarrow \infty . \\
& \Longrightarrow\left\|T y_{n}-T y_{0}\right\|_{1} \longrightarrow 0 \quad \text { as } n \longrightarrow \infty
\end{aligned}
$$

$$
\begin{aligned}
&\left|p(t)\left(T y_{n}^{\prime}(t)-T y_{0}^{\prime}(t)\right)\right|=\mid p(t) \int_{0}^{1} \frac{\partial G(t, s)}{\partial t} q(s)\left[f\left(s, y_{n}(s), p(s) y_{n}^{\prime}(s)\right)\right. \\
&\left.\quad-f\left(s, y_{0}(s), p(s) y_{0}^{\prime}(s)\right)\right] d s \mid \\
& \longrightarrow 0 \quad \text { as } n \longrightarrow \infty \\
& \Longrightarrow\left\|\left(T y_{n}-T y_{0}\right)^{\prime}\right\|_{1} \longrightarrow 0 \quad \text { as } n \longrightarrow \infty
\end{aligned}
$$

From (4.6) and (4.7),

$$
\left\|T y_{n}-T y_{0}\right\| \longrightarrow 0 \quad \text { as } n \longrightarrow \infty
$$

Hence $T: P \rightarrow P$ is a continuous operator. 
Next we prove that $T$ maps every bounded subset of $P$ into relatively compact set. Let $B=\left\{x \in P:\|x\| \leq k_{2}, k_{2}\right.$ is a positive constant $\}$ be any bounded subset of $P$. For $y \in B$,

$$
\begin{aligned}
T y(t) & =\int_{0}^{1} G(t, s) q(s) f\left(s, y(s), p(s) y^{\prime}(s)\right) d s \\
& \leq \max _{t \in[0,1]} \int_{0}^{1} G(t, s) q(s) f\left(s, y(s), p(s) y^{\prime}(s)\right) d s \\
& \leq \max _{(s, u, v) \in[0,1] \times\left[0, k_{2}\right] \times\left[-k_{2}, k_{2}\right]} f(s, u, v) \max _{t \in[0,1]} \int_{0}^{1} G(t, s) q(s) d s \\
& \Longrightarrow T y(t)<\infty .
\end{aligned}
$$

Therefore $T(B)$ is uniformly bounded. Further, equicontinuity of $T(B)$ follows from

$$
\begin{aligned}
\left|T y\left(t_{1}\right)-T y\left(t_{2}\right)\right| & =\left|\int_{0}^{1}\left[\left.G(t, s)\right|_{t=t_{1}}-\left.G(t, s)\right|_{t=t_{2}}\right] q(s) f\left(s, y(s), p(s) y^{\prime}(s)\right) d s\right| \\
& \longrightarrow 0 \text { as } t_{1} \longrightarrow t_{2}, \\
\left|p(t)\left(T y^{\prime}\left(t_{1}\right)-T y^{\prime}\left(t_{2}\right)\right)\right| & =\left|p(t) \int_{0}^{1}\left[\left.\frac{\partial G(t, s)}{\partial t}\right|_{t=t_{1}}-\left.\frac{\partial G(t, s)}{\partial t}\right|_{t=t_{2}}\right] q(s) f\left(s, y(s), p(s) y^{\prime}(s)\right) d s\right| \\
& \leq 2 \max \left|\frac{\partial G(t, s)}{\partial t}\right| \cdot \sup _{t \in[0,1]} p(t) \int_{t_{1}}^{t_{2}} q(s) f\left(s, y(s), p(s) y^{\prime}(s)\right) d s, \\
& \longrightarrow 0 \text { as } t_{1} \longrightarrow t_{2} .
\end{aligned}
$$

Thus from Arzela-Ascoli Theorem, $T(B)$ is relatively compact subset of $P$ and also $T: P \rightarrow P$ is completely continuous.

Next, define functionals $\alpha, \beta, \psi: P \rightarrow[0, \infty)$ such that

$$
\begin{gathered}
\alpha(y)=\sup _{t \in[0,1]}|y(t)|, \\
\beta(y)=\sup _{t \in(0,1]}\left|p(t) y^{\prime}(t)\right|, \\
\psi(y)=\min _{t \in[\eta, 1]}|y(t)| .
\end{gathered}
$$

Clearly, $\alpha, \beta$ are nonnegative continuous convex functionals such that $\|y\|=\max \{\alpha(y), \beta(y)\}$ satisfying (2.3) and (2.4), and $\psi$ is nonnegative concave functional with $\psi(y) \leq \alpha(y)$. 
Let

$$
\begin{gathered}
C=\min _{t \in[\eta, 1]} \int_{\eta}^{1} G(t, s) q(s) d s, \\
L=\sup _{t \in[0,1]} \int_{0}^{1} G(t, s) q(s) d s, \\
N=\sup _{t \in(0,1]}\left|\int_{0}^{1} p(t) \frac{\partial G(t, s)}{\partial t} q(s) d s\right| .
\end{gathered}
$$

Now we state the main results of this work.

Theorem 4.2. Suppose that (E1)-(E3) are satisfied and $f(t, y, z)$ satisfies the following condition.

$\left(\mathrm{H}_{1}\right)$ if there exist real constants $d>0$ and $L_{1}>0$ such that $f(t, y, z)<\min \left\{d / L, L_{1} / N\right\}$ for $(t, y, z) \in\left\{[0,1] \times[0, d] \times\left[-L_{1}, L_{1}\right]\right\}$,

then boundary value problem (1.1)-(1.2) has at least one nonnegative solution $y_{1}$ such that $\sup _{t \in[0,1]}\left|y_{1}(t)\right|<d$ with $\sup _{t \in(0,1]}\left|p(t) y_{1}^{\prime}(t)\right|<L_{1}$.

Theorem 4.3. Suppose that (E1)-(E3) are satisfied. There exist real constants a, $c, d, L_{1}$, and $L_{2}$ with $c \geq a / \lambda=b>a>d>0, L_{2} \geq L_{1}>0$ such that $a / C \leq \min \left\{c / L, L_{2} / N\right\}$ and $f(t, y, z)$ satisfies following conditions.

$\left(\mathrm{H}_{1}\right) f(t, y, z)<\min \left\{d / L, L_{1} / N\right\}$ for $(t, y, z) \in\left\{[0,1] \times[0, d] \times\left[-L_{1}, L_{1}\right]\right\}$,

$\left(\mathrm{H}_{2}\right) f(t, y, z)>a / C$ for $(t, y, z) \in\left\{[\eta, 1] \times[a, a / \lambda] \times\left[-L_{2}, L_{2}\right]\right\}$,

$\left(\mathrm{H}_{3}\right) f(t, y, z) \leq \min \left\{c / L, L_{2} / N\right\}$ for $(t, y, z) \in\left\{[0,1] \times[0, c] \times\left[-L_{2}, L_{2}\right]\right\}$.

Then boundary value problem (1.1)-(1.2) has at least three nonnegative solutions $y_{1}, y_{2}$, and $y_{3}$ in $\bar{P}\left(\alpha^{c}, \beta^{L_{2}}\right)$ such that

$$
\begin{gathered}
\sup _{t \in[0,1]}\left|y_{1}(t)\right|<d, \quad \sup _{t \in(0,1]}\left|p(t) y_{1}^{\prime}(t)\right|<L_{1}, \\
a<\min _{t \in[\eta, 1]}\left|y_{2}(t)\right| \leq \sup _{t \in[0,1]}\left|y_{2}(t)\right| \leq c, \\
\sup _{t \in(0,1]}\left|p(t) y_{2}^{\prime}(t)\right| \leq L_{2}, \quad \sup _{t \in[0,1]}\left|y_{3}(t)\right| \leq \frac{a}{\lambda} \text { with } \sup _{t \in(0,1]}\left|p(t) y_{3}^{\prime}(t)\right| \leq L_{2} .
\end{gathered}
$$


Proof of Theorem 4.2. Let $U=P\left(\alpha^{d}, \beta^{L_{1}}\right)$ be open subset of $P$. We now show that $T(\bar{U}) \subset \bar{U}$. For $y \in \bar{U}$,

$$
\begin{aligned}
\alpha(T y) & =\sup _{t \in[0,1]}|T y(t)| \\
& =\sup _{t \in[0,1]} \int_{0}^{1} G(t, s) q(s) f\left(s, y(s), p(s) y^{\prime}(s)\right) d s, \\
& <\frac{d}{L} \sup _{t \in[0,1]} \int_{0}^{1} G(t, s) q(s) d s<d, \text { from condition }\left(\mathrm{H}_{1}\right)
\end{aligned}
$$

implies that $\alpha(T y)<d$.

Consider that

$$
\begin{aligned}
\beta(T y) & =\sup _{t \in(0,1]}\left|p(t) T y^{\prime}(t)\right|, \\
& =\sup _{t \in(0,1]}\left|p(t) \int_{0}^{1} \frac{\partial G(t, s)}{\partial t} q(s) f\left(s, y(s), p(s) y^{\prime}(s)\right)\right|, \\
& <\frac{L_{1}}{N} \sup _{t \in(0,1]}\left|\int_{0}^{1} p(t) \frac{\partial G(t, s)}{\partial t} q(s) d s\right|, \text { from condition }\left(\mathrm{H}_{1}\right),
\end{aligned}
$$

implies that $\beta(T y)<L_{1}$.

Thus $T(\bar{U}) \subset \bar{U}$. Next, we show that $T$ has no fixed point on $\partial U(=\bar{U} \backslash U)$. On contrary, suppose there exists a fixed point $y(t)$ on $\partial U$ such that $T y(t)=y(t)$. Then from (4.14) and (4.15), $\alpha(y)<d$ and $\beta(y)<L_{1}$, which are not possible. So the operator $T$ has no fixed point on $\partial U$ and from Theorem $2.10 i(T, U, P)=1$. Thus the operator $T$ has at least one fixed point in $U$ and also the boundary value problem (1.1)-(1.2) has at least one nonnegative solution $y_{1}$ such that $\sup _{t \in[0,1]}\left|y_{1}(t)\right|<d$ with $\sup _{t \in(0,1]}\left|p(t) y_{1}^{\prime}(t)\right|<L_{1}$.

Proof of Theorem 4.3. It is easy to see that $\psi(y) \leq \alpha(y)$ for each $y \in \bar{P}\left(\alpha^{c}, \beta^{L_{2}}\right)$. We now show that $T: \bar{P}\left(\alpha^{c}, \beta^{L_{2}}\right) \rightarrow \bar{P}\left(\alpha^{c}, \beta^{L_{2}}\right)$ is well defined. For $y \in \bar{P}\left(\alpha^{c}, \beta^{L_{2}}\right)$,

$$
\begin{aligned}
\alpha(T y) & =\sup _{t \in[0,1]}|T y(t)| \\
& =\sup _{t \in[0,1]} \int_{0}^{1} G(t, s) q(s) f\left(s, y(s), p(s) y^{\prime}(s)\right) d s, \\
& \leq \frac{c}{L} \sup _{t \in[0,1]} \int_{0}^{1} G(t, s) q(s) d s, \text { from assumption }\left(\mathrm{H}_{3}\right), \\
& \leq c .
\end{aligned}
$$




$$
\begin{aligned}
\beta(T y) & =\sup _{t \in(0,1]}\left|p(t) T y^{\prime}(t)\right| \\
& =\sup _{t \in(0,1]}\left|p(t) \int_{0}^{1} \frac{\partial G(t, s)}{\partial t} q(s) f\left(s, y(s), p(s) y^{\prime}(s)\right)\right| \\
& \leq \frac{L_{2}}{N_{t \in(0,1]}} \sup _{0}\left|\int_{0}^{1} p(t) \frac{\partial G(t, s)}{\partial t} q(s) d s\right|, \text { from assumption }\left(\mathrm{H}_{3}\right), \\
& \leq L_{2} .
\end{aligned}
$$

From (4.16) and (4.17),

$$
T(y) \in \bar{P}\left(\alpha^{c}, \beta^{L_{2}}\right) .
$$

Thus, $T: \bar{P}\left(\alpha^{c}, \beta^{L_{2}}\right) \rightarrow \bar{P}\left(\alpha^{c}, \beta^{L_{2}}\right)$ is well defined, and by Lemma 4.1 , it is completely continuous. Now Condition (2) of Theorem 2.11 can be proved by similar manner. Choose $y(t)=a / \lambda \in P, 0 \leq t \leq 1$, then $\alpha(y)=\sup _{t \in[0,1]}|y(t)|=a / \lambda, \beta(y)=\sup _{t \in(0,1]}\left|p(t) y^{\prime}(t)\right|=0<$ $L_{2}, \psi(x)=\min _{t \in[\eta, 1]}|y(t)|=a / \lambda>a$. Thus, $\left\{y \in \bar{P}\left(\alpha^{b=a / \lambda}, \beta^{L_{2}}, \Psi_{a}\right): \Psi(y)>a\right\} \neq \Phi$. Further if $y \in \bar{P}\left(\alpha^{a / \lambda}, \beta^{L_{2}}, \Psi_{a}\right)$, then $a \leq y(t) \leq a / \lambda$ for $\eta \leq t \leq 1$. Then by definition of $\psi$ and assumption $\left(\mathrm{H}_{2}\right)$, we have

$$
\begin{aligned}
\psi(T y) & =\min _{t \in[\eta, 1]}|T y(t)| \\
& =\min _{t \in[\eta, 1]} \int_{0}^{1} G(t, s) q(s) f\left(s, y(s), p(s) y^{\prime}(s)\right) d s \\
& \geq \min _{t \in[\eta, 1]} \int_{\eta}^{1} G(t, s) q(s) f\left(s, y(s), p(s) y^{\prime}(s)\right) d s \\
& >\frac{a}{C} \cdot C=a
\end{aligned}
$$

Thus, Condition (1) of Theorem 2.11 is satisfied. We finally show that condition (3) of Theorem 2.11 holds, too. Suppose $y \in \bar{P}\left(\alpha^{c}, \beta^{L_{2}}, \Psi_{a}\right)$ with $\alpha(T y)>b$. Then by definition of $\psi$ and $T y \in P$, we have

$$
\begin{aligned}
\Psi(T y) & =\min _{t \in[\eta, 1]}|T y(t)| \\
& \geq \lambda \max _{t \in[0,1]}|T y(t)| \\
& \geq \curlywedge \alpha(T y) \\
& =a .
\end{aligned}
$$


So, Condition (3) of Theorem 2.11 is also satisfied. Therefore, Theorem 2.11 yields that boundary value problem (1.1)-(1.2) has at least three nonnegative solutions $y_{1}, y_{2}$, and $y_{3}$ in $\bar{P}\left(\alpha^{c}, \beta^{L_{2}}\right)$ such that

$$
\begin{gathered}
\sup _{t \in[0,1]}\left|y_{1}(t)\right|<d, \quad \sup _{t \in(0,1]}\left|p(t) y_{1}^{\prime}(t)\right|<L_{1} ; \\
a<\min _{t \in[\eta, 1]}\left|y_{2}(t)\right| \leq \sup _{t \in[0,1]}\left|y_{2}(t)\right| \leq c, \quad \sup _{t \in(0,1]}\left|p(t) y_{2}^{\prime}(t)\right| \leq L_{2} ; \\
\sup _{t \in[0,1]}\left|y_{3}(t)\right| \leq \frac{a}{\lambda^{\prime}}, \quad \sup _{t \in(0,1]}\left|p(t) y_{3}^{\prime}(t)\right| \leq L_{2} .
\end{gathered}
$$

Corollary 4.4. Suppose that (E1)-(E3) are satisfied. If there exist constants $0<d_{1}<a_{1}<$ $a_{1} / \lambda<d_{2}<a_{2}<a_{2} / \lambda<d_{3}<\cdots<d_{n}, 0<L_{1} \leq L_{2} \leq L_{3} \leq \cdots \leq L_{n} n \in \mathbb{N}$, with $a_{i} / C<\min \left\{d_{i+1} / L, L_{i+1} / N\right\}, 1<i \leq n-1$ such that $f$ satisfies the following conditions:

(M1) $f(t, y, z)<\min \left\{d_{i} / L, L_{i} / N\right\}$, for $(t, y, z) \in\left\{[0,1] \times\left[0, d_{i}\right] \times\left[-L_{i}, L_{i}\right]\right\}, 1 \leq i \leq n$,

(M2) $f(t, y, z)>a_{i} / C$, for $(t, y, z) \in\left\{[\eta, 1] \times\left[a_{i}, a_{i} / \lambda\right] \times\left[-L_{i+1}, L_{i+1}\right]\right\}, 1 \leq i \leq n-1$,

then boundary value problem (1.1)-(1.2) has at least $2 n-1$ nonnegative solutions.

Proof. When $n=1$, the result follows from Theorem 4.2. When $n=2$, it is clear that all the conditions of Theorem 4.3 hold (with $c=d_{2}, d=d_{1}, a=a_{1}$ ). Thus the boundary value problem (1.1)-(1.2) has at least three positive solutions $y_{1}, y_{2}$, and $y_{3}$. Following this way, we complete the proof by induction method.

Finally, we demonstrate these results through examples.

\section{Example}

In Example 5.1, we demonstrate the detailed working of the boundary value problem (1.5) mentioned in the introduction. Example 5.2 verifies our results.

Example 5.1. Consider the boundary value problem (1.5).

Here,

$$
f\left(t, y, p y^{\prime}\right)=2(r+1) t-\frac{5}{4} r, \quad q(t)=t^{r-1}, \quad \max _{t \in[0,1]} f\left(t, y, p y^{\prime}\right)=\frac{3}{4} r+2
$$

Following the notations of this work, it is easy to see that

$$
L=\frac{3}{2}\left[\frac{3(1-r)}{4-4^{r}}\right]^{(1-r) / r}, \quad N=\frac{3(1-r)}{r\left(4-4^{r}\right)}
$$

Now for $d \geq(3 / 2)\left[3(1-r) / 4-4^{r}\right]^{(1-r) / r}[(3 / 4) r+2]$ and $L_{1} \geq 3(1-r)((3 / 4) r+1) / r(4-$ $\left.4^{r}\right), f\left(t, y, p y^{\prime}\right) \leq \min \left\{d / L, L_{1} / N\right\}$. 
Then from Theorem 4.2, the problem has at least one nonnegative solution $y(t)$ with

$$
\sup _{t \in[0,1]}|y(t)| \leq \frac{3}{2}\left[\frac{3(1-r)}{4-4^{r}}\right]^{(1-r) / r}\left[\frac{3}{4} r+2\right], \quad \sup _{t \in(0,1]}\left|p(t) y^{\prime}(t)\right| \leq \frac{3(1-r)}{r\left(4-4^{r}\right)}\left[\frac{3}{4} r+2\right] \text {. }
$$

Next we reduce the problem and then apply Theorem 4.2 for $p(t)=1$. Using the transformation $x=(1-r) \int_{0}^{t}\left(1 / x^{r}\right) d x=t^{1-r}$, the boundary value problem (1.5) can be reduced to regular boundary value problem as

$$
\begin{gathered}
-v^{\prime \prime}(x)=\frac{1}{(1-r)^{2}} x^{(2 r-1) /(1-r)}\left[2(r+1) x^{1 /(1-r)}-\frac{5}{4} r\right], \quad x \in(0,1), \\
v(0)=0, \quad v(1)=v\left[\left(\frac{1}{4}\right)^{(1-r)}\right] .
\end{gathered}
$$

Here,

$$
\begin{gathered}
F\left(x, v, v^{\prime}\right)=\frac{1}{(1-r)^{2}} t^{(2 r-1) /(1-r)}\left[2(r+1) t^{1 /(1-r)}-\frac{5}{4} r\right] \\
\max _{x \in[0,1]} F\left(x, v, v^{\prime}\right)=\frac{1}{(1-r)^{2}}\left[2(1+r)-\frac{5}{4} r\right]
\end{gathered}
$$

Now following the notation of this work for $p(t)=1$,

$$
L=\frac{3(1-r)^{2}}{4-4^{r}}\left[\frac{3(1-r)}{r\left(4-4^{r}\right)}\right]^{(1-r) / r}, \quad N=\frac{3(1-r)^{2}}{r\left(4-4^{r}\right)} .
$$

Now for $d \geq 3 /\left(4-4^{r}\right)\left[3(1-r) /\left(4-4^{r}\right)\right]^{(1-r) / r}[(3 / 4) r+2]$ and $L_{1} \geq\left(3 / r\left(4-4^{r}\right)\right)[(3 / 4) r+$ $2], f\left(t, v, v^{\prime}\right) \leq \min \left\{d / L, L_{1} / N\right\}$. So the problem has at least one nonnegative solution $v(t)$ with

$$
\sup _{t \in[0,1]}|v(t)| \leq \frac{3}{4-4^{r}}\left[\frac{3(1-r)}{4-4^{r}}\right]^{(1-r) / r}\left[\frac{3}{4} r+2\right], \quad \sup _{t \in(0,1]}\left|v^{\prime}(t)\right| \leq \frac{3}{r\left(4-4^{r}\right)}\left[\frac{3}{4} r+2\right] .
$$

Hence the boundary value problem (1.5) has at least one nonnegative solution $y(t)$ with

$$
\sup _{t \in[0,1]}|y(t)| \leq \frac{3}{4-4^{r}}\left[\frac{3(1-r)}{4-4^{r}}\right]^{(1-r) / r}\left[\frac{3}{4} r+2\right], \quad \sup _{t \in(0,1]}\left|y^{\prime}(t)\right| \leq \frac{3}{r\left(4-4^{r}\right)}\left[\frac{3}{4} r+2\right] \text {. }
$$

Now in this case it is easy to show that if $r$ approaches one, that is, the order of singularity increases, upper bound for $\sup _{t \in[0,1]}|y(t)|$ approaches $\infty$ while in case of direct solving, upper bound for $\sup _{t \in[0,1]}|y(t)|$ approaches 4.125. As smaller upper bound for $\sup _{t \in[0,1]}|y(t)|$ will enable to find nonnegative solution(s) faster and hence will be helpful in constructing efficient numerical algorithms to find multiple nonnegative solutions. 
Example 5.2. Consider the following boundary value problem:

$$
\begin{gathered}
-\left(t^{1 / 2} y^{\prime}(t)\right)^{\prime}=t^{-1 / 2} f\left(t, y(t), t^{1 / 2} y^{\prime}(t)\right), \quad 0<t<1, \\
y(0)=0, \quad y(1)=\frac{3}{2} y\left(\frac{1}{3}\right) .
\end{gathered}
$$

(i) If

$$
f(t, y, z)= \begin{cases}\frac{\sin t}{3}+\frac{10 y^{2}+8}{400}+\left(\frac{z}{300}\right)^{2}, & y \leq 4 \\ \frac{\sin t}{3}+\frac{21}{50}+\left(\frac{z}{300}\right)^{2}, & y \geq 4\end{cases}
$$

then the boundary value problem (5.9) has at least one nonnegative solution.

(ii) Further, if

$$
f(t, y, z)= \begin{cases}\frac{\sin t}{3}+\frac{10 y^{2}+8}{400}+\left(\frac{z}{4000}\right)^{2}, & y \leq 4 \\ \frac{\sin t}{3}+\frac{9 y^{2}+24}{400}+\left(\frac{z}{4000}\right)^{2}, & 4 \leq y \leq 191 \\ \frac{\sin t}{3}+\frac{328353}{400}+\left(\frac{z}{4000}\right)^{2}, & y \geq 191\end{cases}
$$

then the boundary value problem (5.9) has at least three nonnegative solutions.

Proof. Here, $\alpha_{1}=3 / 2$ and $\eta=1 / 3$. After simple calculation, we get $L=5.464, N=3.732$, $C=1.5396$, and $\lambda=0.1786$.

(i) At least one nonnegative solution: we choose $d=3$ and $L_{1}=10$. Here, $\min \left\{d / L, L_{1} / N\right\}=0.549$;

$$
f(t, y, z)<0.549, \quad \text { for } 0 \leq t \leq 1,0 \leq y \leq 3,-10 \leq z \leq 10 \text {. }
$$

Thus, condition $\left(H_{1}\right)$ is satisfied. Now from Theorem 4.2 the problem has at least one nonnegative solution $y_{1}$ such that $\sup _{t \in[0,1]}\left|y_{1}(t)\right|<3$ with $\sup _{t \in(0,1]}\left|p(t) y_{1}^{\prime}(t)\right|<10$.

(ii) At least three nonnegative solutions: we choose constants $d=4, a=34, c=4800$, $L_{1}=10$, and $L_{2}=3500$. Here, $\min \left\{d / L, L_{1} / N\right\}=0.732, \min \left\{c / L, L_{2} / N\right\}=878.45, a / \lambda=$ 190.34, and $a / C=22.084$;

$$
\begin{gathered}
f(t, y, z)<0.732, \quad \text { for } 0 \leq t \leq 1,0 \leq y \leq 4,-10 \leq z \leq 10 \\
f(t, y, z)>22.084 \text { for } \frac{1}{3} \leq t \leq 1,34 \leq y \leq 190.34,-3500 \leq z \leq 3500 \\
f(t, y, z)<878.45 \text { for } 0 \leq t \leq 1,0 \leq y \leq 4800,-3500 \leq z \leq 3500 .
\end{gathered}
$$


Thus, conditions $\left(H_{1}\right),\left(H_{2}\right)$, and $\left(H_{3}\right)$ are satisfied. Now from Theorem 4.3 the problem has at least three nonnegative solutions $y_{1}, y_{2}$, and $y_{3}$ such that

$$
\begin{gathered}
\sup _{t \in[0,1]}\left|y_{1}(t)\right|<4, \quad \sup _{t \in(0,1]}\left|p(t) y_{1}(t)\right|<10 ; \\
34<\min _{t \in[\eta, 1]}\left|y_{2}(t)\right| \leq \sup _{t \in[0,1]}\left|y_{2}(t)\right|<4800, \quad \sup _{t \in(0,1]}\left|p(t) y_{2}^{\prime}(t)\right| \leq 3500, \\
\sup _{t \in[0,1]}\left|y_{3}(t)\right| \leq 190.34, \quad \sup _{t \in(0,1]}\left|p(t) y_{3}^{\prime}(t)\right| \leq 3500 .
\end{gathered}
$$

Remark 5.3. For $\alpha_{1}=1$, the problem (1.1)-(1.2) can be regarded as two-point boundary value problem with boundary conditions as

$$
y(0)=0, \quad y^{\prime}(1)=0,
$$

in the limiting case $\eta \rightarrow 1^{-}$. Thus the results established in this work are also valid for the two-point boundary value problem [21,22].

Remark 5.4. Theorem 4.3 and Corollary 4.4 extend Theorem 3.1 and Corollary 3.1 of [19] to doubly singular three-point boundary value problem.

\section{Acknowledgments}

This work is supported by DST, New Delhi, and UGC, New Delhi, India.

\section{References}

[1] L. E. Bobisud, "Existence of solutions for nonlinear singular boundary value problems," Applicable Analysis. An International Journal, vol. 35, no. 1-4, pp. 43-57, 1990.

[2] Y. Zou, Q. Hu, and R. Zhang, "On numerical studies of multi-point boundary value problem and its fold bifurcation," Applied Mathematics and Computation, vol. 185, no. 1, pp. 527-537, 2007.

[3] M. S. Berger and L. E. Fraenkel, "Nonlinear desingularization in certain free-boundary problems," Communications in Mathematical Physics, vol. 77, no. 2, pp. 149-172, 1980.

[4] R. A. Khan, "Quasilinearization method and nonlocal singular three point boundary value problems," Electronic Journal of Qualitative Theory of Differential Equations, no. Special Edition I, p. No. 17, 13, 2009.

[5] M. Moshinsky, "Sobre los problemas de condiciones a la frontiera en una dimension de caracteristicas discontinuas," Boletin Sociedad Matemática Mexicana, vol. 7, pp. 1-25, 1950.

[6] C. P. Gupta, "Solvability of a three-point nonlinear boundary value problem for a second order ordinary differential equation," Journal of Mathematical Analysis and Applications, vol. 168, no. 2, pp. 540-551, 1992.

[7] X. He and W. Ge, "Triple solutions for second-order three-point boundary value problems," Journal of Mathematical Analysis and Applications, vol. 268, no. 1, pp. 256-265, 2002.

[8] B. Liu, "Positive solutions of a nonlinear three-point boundary value problem," Computers $\mathcal{E}$ Mathematics with Applications, vol. 44, no. 1-2, pp. 201-211, 2002.

[9] B. Liu, L. Liu, and Y. Wu, "Positive solutions for singular second order three-point boundary value problems," Nonlinear Analysis, vol. 66, no. 12, pp. 2756-2766, 2007.

[10] R. Ma, "Positive solution of nonlinear three-point boundary value problems," Electronic Journal of Differential Equations, vol. 1999, pp. 1-8, 1999. 
[11] R. Ma, "Positive solutions for second-order three-point boundary value problems," Applied Mathematics Letters, vol. 14, no. 1, pp. 1-5, 2001.

[12] J. R. L. Webb, "Positive solutions of some three point boundary value problems via fixed point index theory," in Proceedings of the Third World Congress of Nonlinear Analysts, Part 7 (Catania, 2000), vol. 47, no. 7, pp. 4319-4332, 2001.

[13] Z. Du, C. Xue, and W. Ge, "Multiple solutions for three-point boundary value problem with nonlinear terms depending on the first order derivative," Archiv der Mathematik, vol. 84, no. 4, pp. 341-349, 2005.

[14] R. A. Khan and J. R. L. Webb, "Existence of at least three solutions of a second-order three-point boundary value problem," Nonlinear Analysis: Theory, Methods E Applications, vol. 64, no. 6, pp. 13561366, 2006.

[15] Y. Guo and W. Ge, "Positive solutions for three-point boundary value problems with dependence on the first order derivative," Journal of Mathematical Analysis and Applications, vol. 290, no. 1, pp. 291-301, 2004.

[16] J. Henderson, “Uniqueness implies existence for three-point boundary value problems for second order differential equations," Applied Mathematics Letters, vol. 18, no. 8, pp. 905-909, 2005.

[17] Y. Chen, B. Yan, and L. Zhang, "Positive solutions for singular three-point boundary-value problems with sign changing nonlinearities depending on $x^{\prime}, "$ Electronic Journal of Differential Equations, p. 1-9, 2007.

[18] R. P. Agarwal, D. O'regan, and B. Yan, "Positve solutions for singular three-point bound-ary value problems," Electronic Journal of Differential Equations, vol. 2008, pp. 1-20, 2008.

[19] Z. Bai and W. Ge, "Existence of three positive solutions for some second-order boundary value problems," Computers \& Mathematics with Applications, vol. 48, no. 5-6, pp. 699-707, 2004.

[20] D. J. Guo and V. Lakshmikantham, Nonlinear problems in abstract cones, vol. 5 of Notes and Reports in Mathematics in Science and Engineering, Academic Press, Boston, MA, 1988.

[21] R. Ma, "A survey on nonlocal boundary value problems," Applied Mathematics E-Notes, vol. 7, pp. 257-279, 2007.

[22] W. Feng and J. R. L. Webb, "Solvability of $m$-point boundary value problems with nonlinear growth," Journal of Mathematical Analysis and Applications, vol. 212, no. 2, pp. 467-480, 1997. 


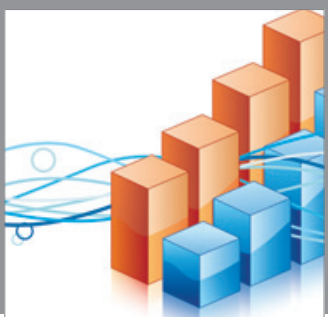

Advances in

Operations Research

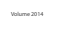

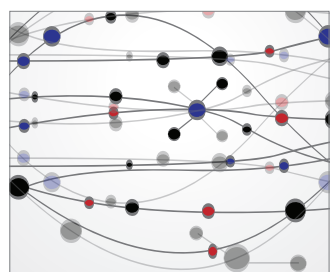

\section{The Scientific} World Journal
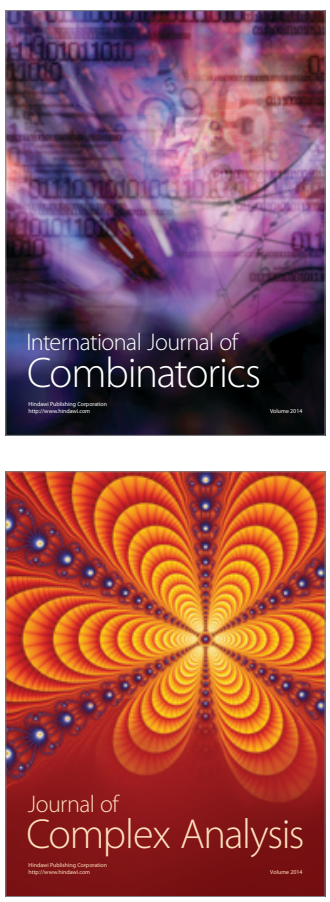

International Journal of

Mathematics and

Mathematical

Sciences
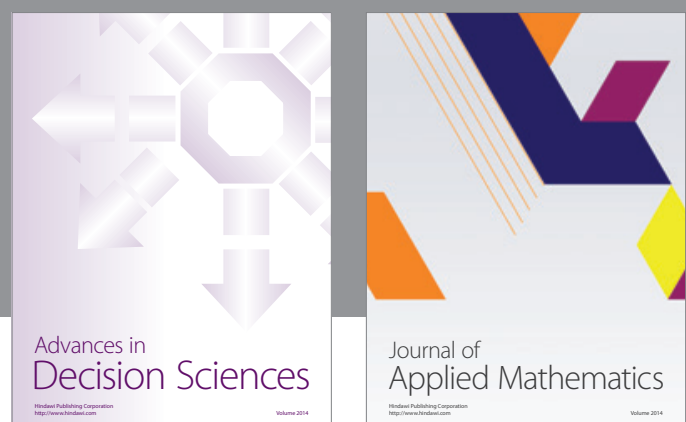

Journal of

Applied Mathematics
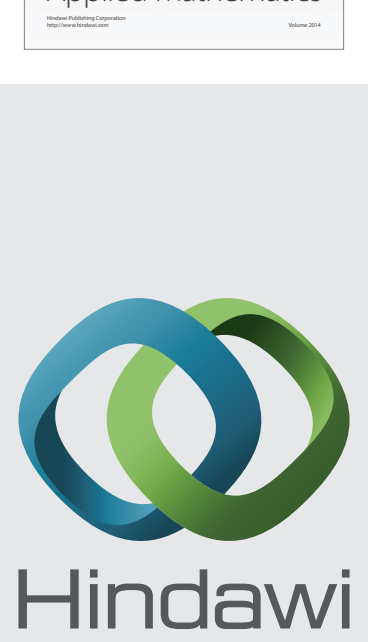

Submit your manuscripts at http://www.hindawi.com
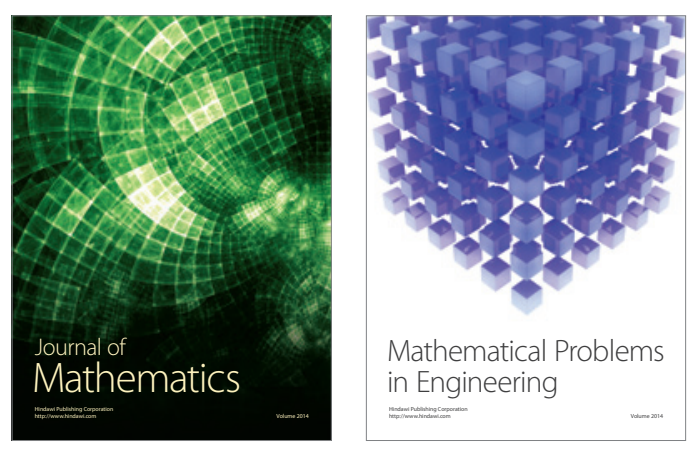

Mathematical Problems in Engineering
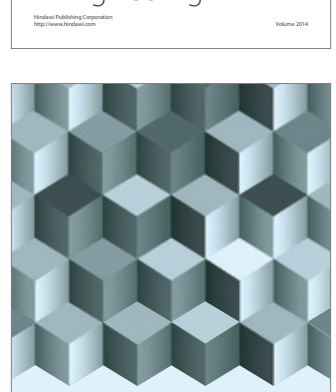

Journal of

Function Spaces
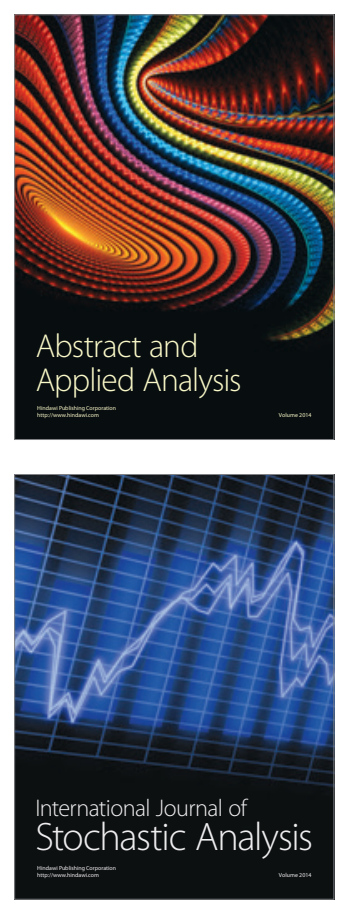

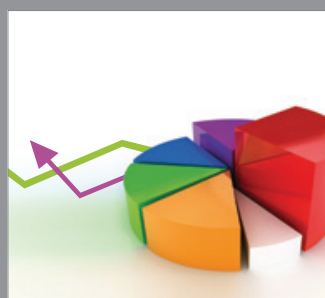

ournal of

Probability and Statistics

Promensencen
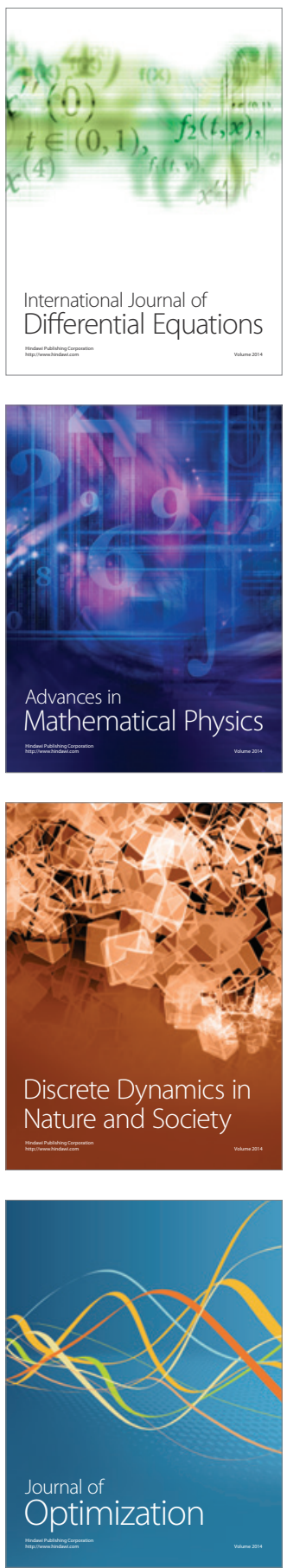